\title{
Uso de denosumab para el quiste óseo aneurismático de columna. Reporte de un caso y revisión bibliográfica
}

\author{
Felipe Lanari Zubiaur, ${ }^{\star}$ Alfredo O. Godoy Adaro," Pedro L. Bazán" \\ "Grupo Médico Vertebral, Ciudad Autónoma de Buenos Aires, Argentina \\ ** Clínica Modelo S. A., Paraná, Entre Ríos, Argentina \\ "Hospital Interzonal General de Agudos "General San Martín”, La Plata, Buenos Aires, Argentina
}

\begin{abstract}
RESUMEN
Los tumores vertebrales de columna tienen una frecuencia muy baja. La incidencia de quiste óseo aneurismático es del 1-4\% de todos los tumores. Su ubicación predominante es la columna lumbar, en mayor medida, en el arco posterior. Según estudios publicados, esta enfermedad se comporta de forma agresiva, por la compresión radicular que genera debido a su expansión. Si bien hay bibliografía sobre el tratamiento quirúrgico del tumor y su resección en bloque y embolización, no hay experiencias sobre el tratamiento con anticuerpos monoclonales. Existen muy pocos artículos que corroboren un tratamiento para las recidivas o los quistes óseos aneurismáticos inoperables o intralesionales, los cuales dejan la posibilidad abierta a otras técnicas. Presentamos el caso de una mujer de 38 años con lumbocruralgia. En los estudios, se detecta una tumoración, y la biopsia por punción y las imágenes confirman que se trata de un quiste óseo aneurismático. Se planifica el tratamiento con embolización preoperatoria y exéresis intracompartimental mediante cirugía posterior con liberación del saco dural. A los seis meses, el tumor recidiva. Se indica tratamiento con denosumab por dos años, se realizan controles periódicos y se constata la remisión de los síntomas y la mejoría de las imágenes.
\end{abstract}

Palabra clave: Quiste óseo aneurismático; tratamiento; recidiva; coadyuvante; denosumab.

Nivel de Evidencia: IV

\section{Use of Denosumab in an Aneurysmal Bone Cyst of the Spine. Case Report and Literature Review}

\section{ABSTRACT}

Vertebral tumors of the spine have a very low frequency. The incidence of aneurysmal bone cyst $(\mathrm{ABC})$ ranges between $1 \%$ and $4 \%$ of all tumors. Its predominant location is the lumbar spine, to a greater extent, in the posterior arch. According to published studies, this condition behaves aggressively, due to the root compression generated by its expansion. While there is literature on the surgical treatment of the tumor and its en bloc resection and embolization, there is no experience on the treatment with monoclonal antibodies. There are very few published articles that corroborate a treatment for recurrences or for inoperable or intralesional aneurysmal bone cysts, which leave the possibility open to other techniques. We present the case of a 38-year-old woman with lumbar pain radiating to the thigh. In studies, a tumor was detected, and the needle biopsy and imaging confirmed that it was an aneurysmal bone cyst. We planned the treatment with preoperative embolization and intracompartmental excision by subsequent surgery with the release of the dural sac. At six months, tumor recurrence was observed. Treatment with denosumab was indicated for two years, periodic controls were carried out and the remission of symptoms and improvement in the images were verified.

Key words: Aneureysmal bone cyst; treatment; relapse; coadjuvant; denosumab.

Level of Evidence: IV 


\section{INTRODUCCIÓN}

Los tumores vertebrales en la columna tienen una frecuencia muy baja, el 1-4\% corresponde al quiste óseo aneurismático (QOA). ${ }^{1-10}$ El QOA es un tumor benigno expansivo. Si bien se consideró un seudotumor por muchos años, actualmente, se ha demostrado que es un tumor benigno asociado a un patrón específico alterado en el gen USP6 localizado en el cromosoma 17p13.,

En el 70\% de los pacientes, el QOA se detecta como presentación única y, en el 30\%, asociado a otros tumores. Es frecuente en la columna lumbar con predominio en el arco posterior. Entre sus características más distintivas, se mencionan el crecimiento lento, con islotes sanguíneos separados por tabiques conectivos, ${ }^{1,2,6-8}$ y un comportamiento agresivo por la compresión radicular resultante de su expansión. . $^{6,7}$,

Desde el punto de vista histológico, los QOA se caracterizan por tener células gigantes multinucleadas, células de estroma, originado de células osteoblásticas mesenquimatosas. Es un tumor benigno agresivo, ${ }^{1-3,6,10}$ que, según la clasificación de Enneking, varía de los estadios 1 a 3.,

Los pacientes presentan, en general, dolor y rigidez, si aparece en la columna, los síntomas pueden ser agresivos por la compresión medular o radicular, las fracturas patológicas por la extensión tumoral, la inestabilidad o el déficit neurológico. ${ }^{6,7,9}$

Los tratamientos para el QOA en la columna lumbar y la pelvis han sido un desafío quirúrgico, ya que su abordaje y sus complicaciones no han dejado de ser un problema para tratarlos definitivamente. Si bien la primera línea de abordaje ha sido la embolización arterial selectiva y la resección en bloque, ${ }^{1-3,6-8,10}$ se han instaurado técnicas coadyuvantes para tratar pacientes que no son candidatos a las embolizaciones, tienen tumores irresecables o que recidivan luego de estos tratamientos. ${ }^{2,3,6,7,9}$ Una de las posibles opciones terapéuticas coadyuvantes es el uso de denosumab, un anticuerpo monoclonal totalmente humano que inhibe el ligando RANK (receptor activator of nuclear factor kappa-B ligand) y cuyo efecto es disminuir drásticamente la resorción ósea en tumores de estas características, que comparte con el tumor de células gigantes. ${ }^{2,3,7-9,11}$

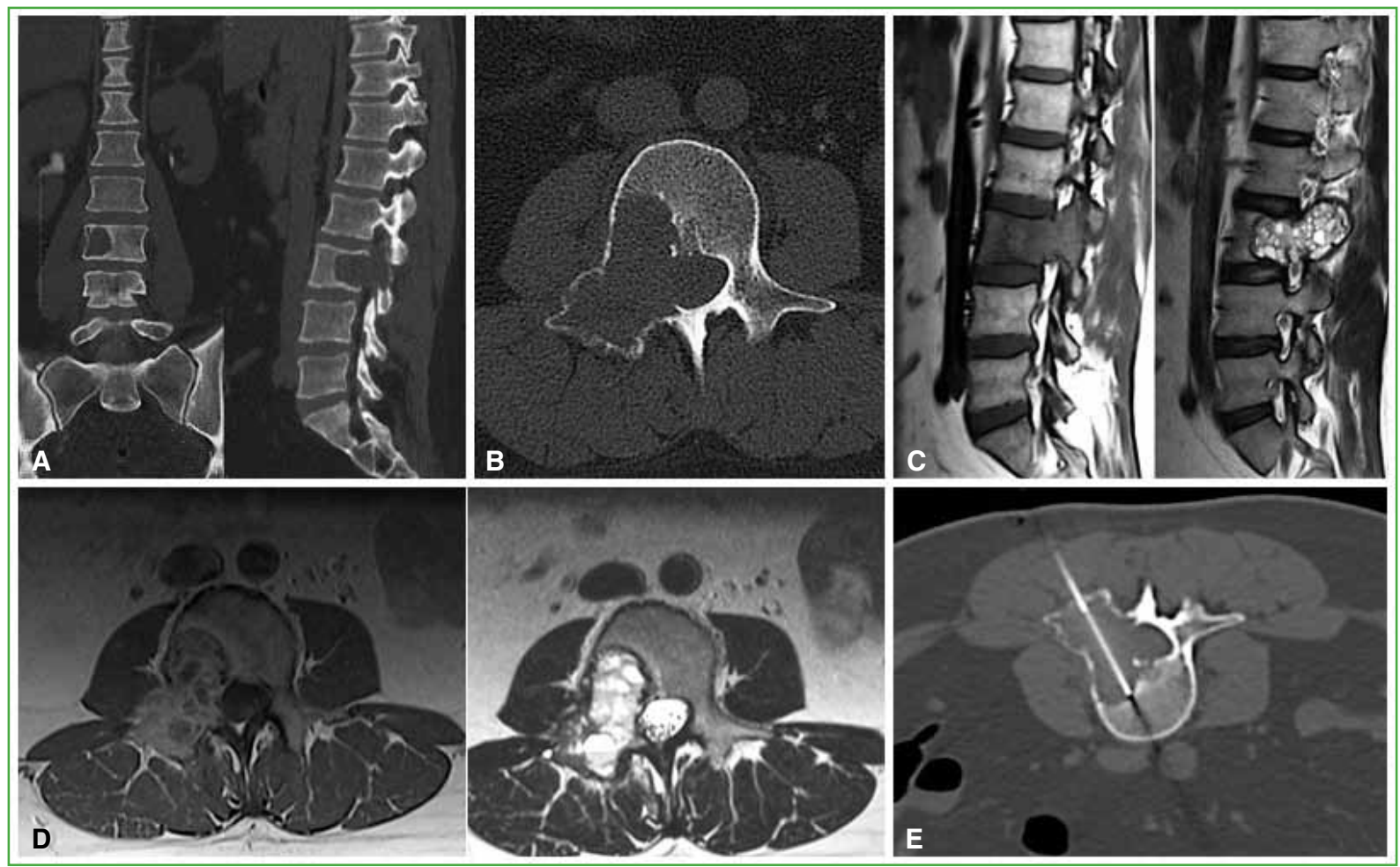

Figura 1. Estudios iniciales. Tomografía multicorte de columna lumbosacra. A. Cortes coronal y sagital. B. Corte axial que muestra una lesión de frágil pared en la pared medial del pedículo. Resonancia magnética de columna lumbosacra. C. Corte sagital en secuencias T1 y T2. Se observa la lesión quística que compromete el cuerpo e insufla pedículo y facetas articulares. D. Corte axial en secuencias T1 y T2. Se visualiza el compromiso del canal. E. Imagen tomográfica de la punción biopsia con aguja fina. 
Presentamos este caso con el propósito de examinar el uso de denosumab en pacientes con recidiva del QOA, conocer el efecto de la dosis en el tiempo y la posibilidad de realizar una cirugía después del tratamiento.

\section{CASO CLÍNICO}

Mujer de 38 años que acudió a la consulta con dolor lumbocrural a predominio derecho que fue aumentando con el tiempo, no tenía déficit motor, las caderas estaban libres, la maniobra de Lasegue fue negativa, la de Wasserman, positiva a predominio derecho. Se detectó hiporreflexia rotuliana bilateral con hiperestesia superficial y profunda de L3 y L4 derecho, pie cavo varo y pulsos positivos bilaterales. Refirió litiasis biliar. Concurrió con un centellograma óseo corporal total y una tomografía computarizada por emisión de fotón único (SPECT) con 20/30 mCi de $\mathrm{TC}^{99}$ que informaban una lesión única en L3; una tomografía previa de tórax, abdomen y pelvis no revelaba hallazgos de metástasis o algún otro foco tumoral posible, antes de la punción para determinar el tipo y la estirpe de la patología en cuestión; y una mamografía BI-RADS 2 que no mostraba enfermedad tumoral.

Se solicitaron otros estudios por imágenes, tomografía helicoidal y resonancia magnética, y se confirmó la ubicación de la lesión dentro de la vértebra. La biopsia por punción guiada por tomografía (Figura 1) confirmó el diagnóstico de QOA. A los dos meses de la consulta y los estudios, el quiste tuvo una evolución tórpida y los síntomas se incrementaron; por lo tanto, se solicitaron nuevos estudios en los que se observó la progresión e inclusive una fractura patológica en el cuerpo de L3 (Figura 2).

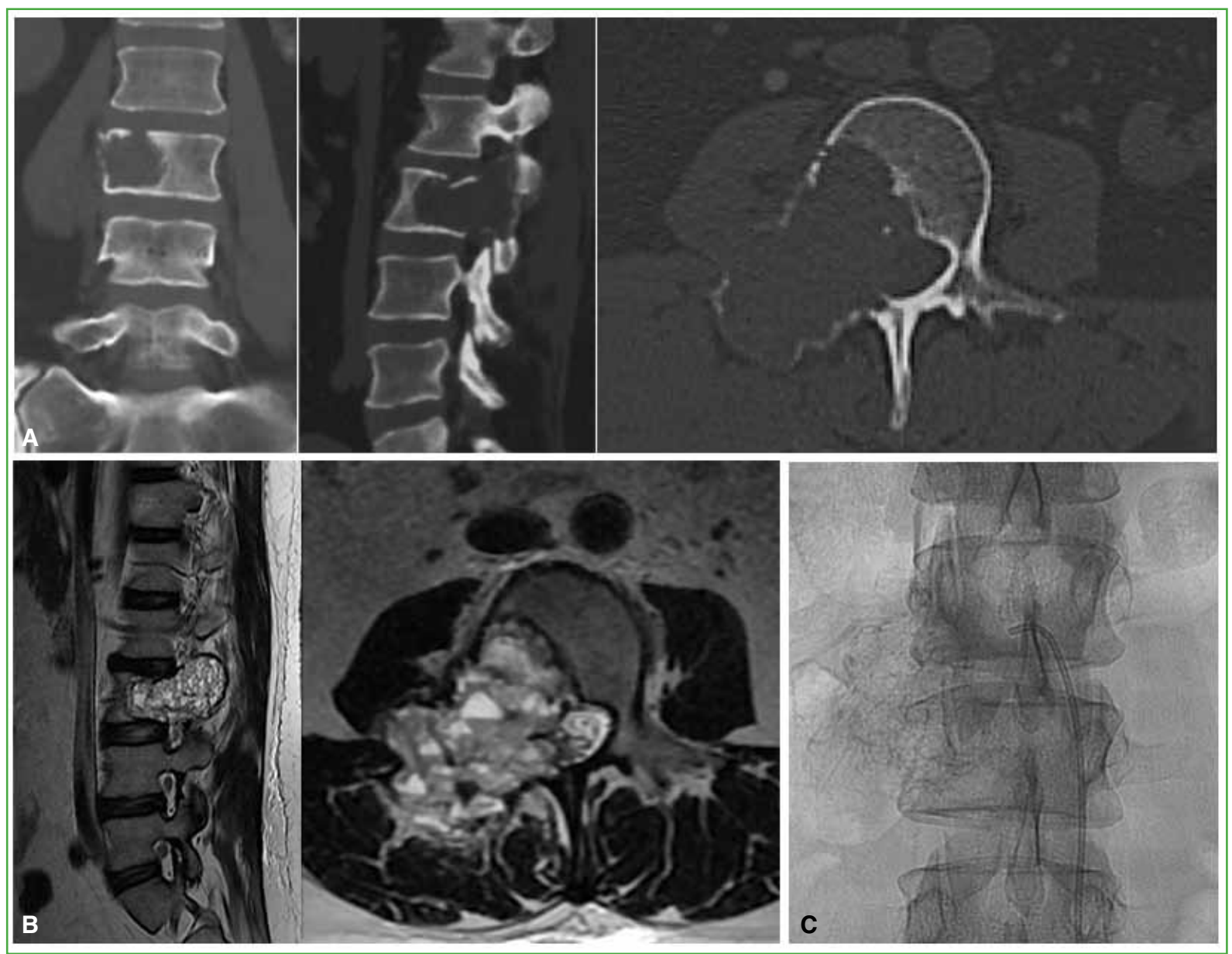

Figura 2. Estudios complementarios a los dos meses del diagnóstico que muestran la progresión de la enfermedad. A. Tomografía multicorte de columna lumbosacra, cortes coronal, sagital y axial. B. Resonancia magnética de columna lumbosacra, cortes sagital y axial. C. Embolización radioscópica preoperatoria. 
Se decidió evaluar las posibilidades de tratamiento para esta paciente y los puntos considerados fueron: que era una lesión benigna rápidamente progresiva, tipo G0III (agresivo) extracapsular y extracompartimental M0, según la clasificación de Enneking; la paciente no tenía síntomas de inestabilidad potencial en el momento del examen y sí presentaba trastornos neurológicos progresivos. Después de analizar la bibliografía, se optó por la embolización previa selectiva, $24 \mathrm{~h}$ antes de la intervención, un abordaje por vía posterior, la exéresis de la lesión intracompartimental con fenol como sustancia coadyuvante y fresado de la lesión, la liberación del saco dural y de las raíces de L2 y también de L3 con artrodesis de las vértebras L2, L3 y L4 (Figura 3).

La evolución posoperatoria fue buena.

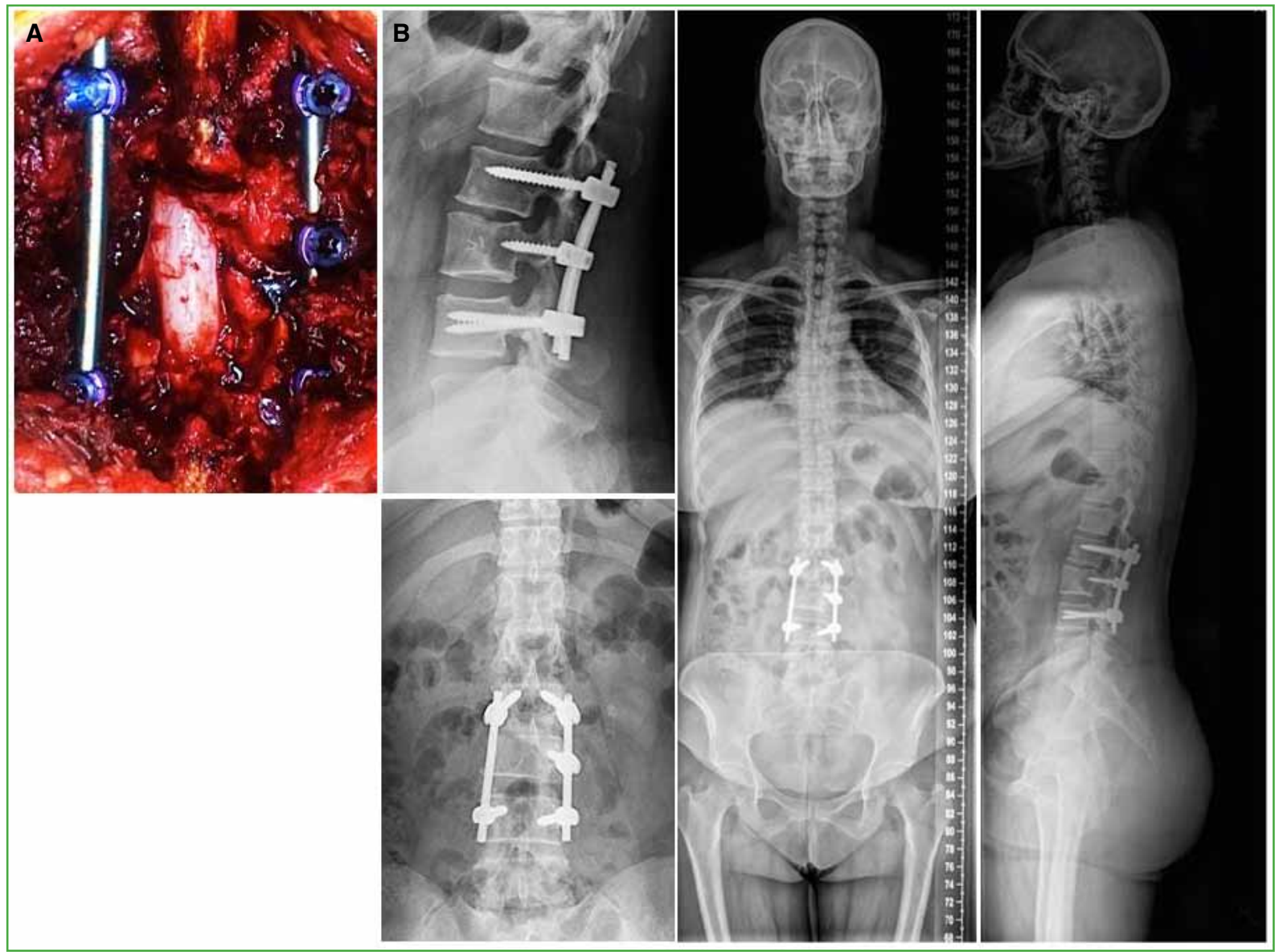

Figura 3. A. Imagen intraoperatoria. B. Radiografías y espinograma posoperatorios. 
A los seis meses del procedimiento, la paciente no tiene síntomas. La tomografía computarizada y la resonancia magnética de control (Figura 4) muestran una imagen compatible con recidiva local del QOA. Después de consultar la bibliografía citada en este artículo, se discutió el nuevo tratamiento con las siguientes opciones: nueva vertebrectomía de L3, inyecciones intratumorales de corticoides y calcitonina, embolización del tumor, administración de denosumab. Se optó por el tratamiento con denosumab $120 \mathrm{mg}$, dosis inicial, cada tres meses, por dos años. A medio término se realizó un control tomográfico y se constató la remisión y la calcificación de la lesión (Figura 5).
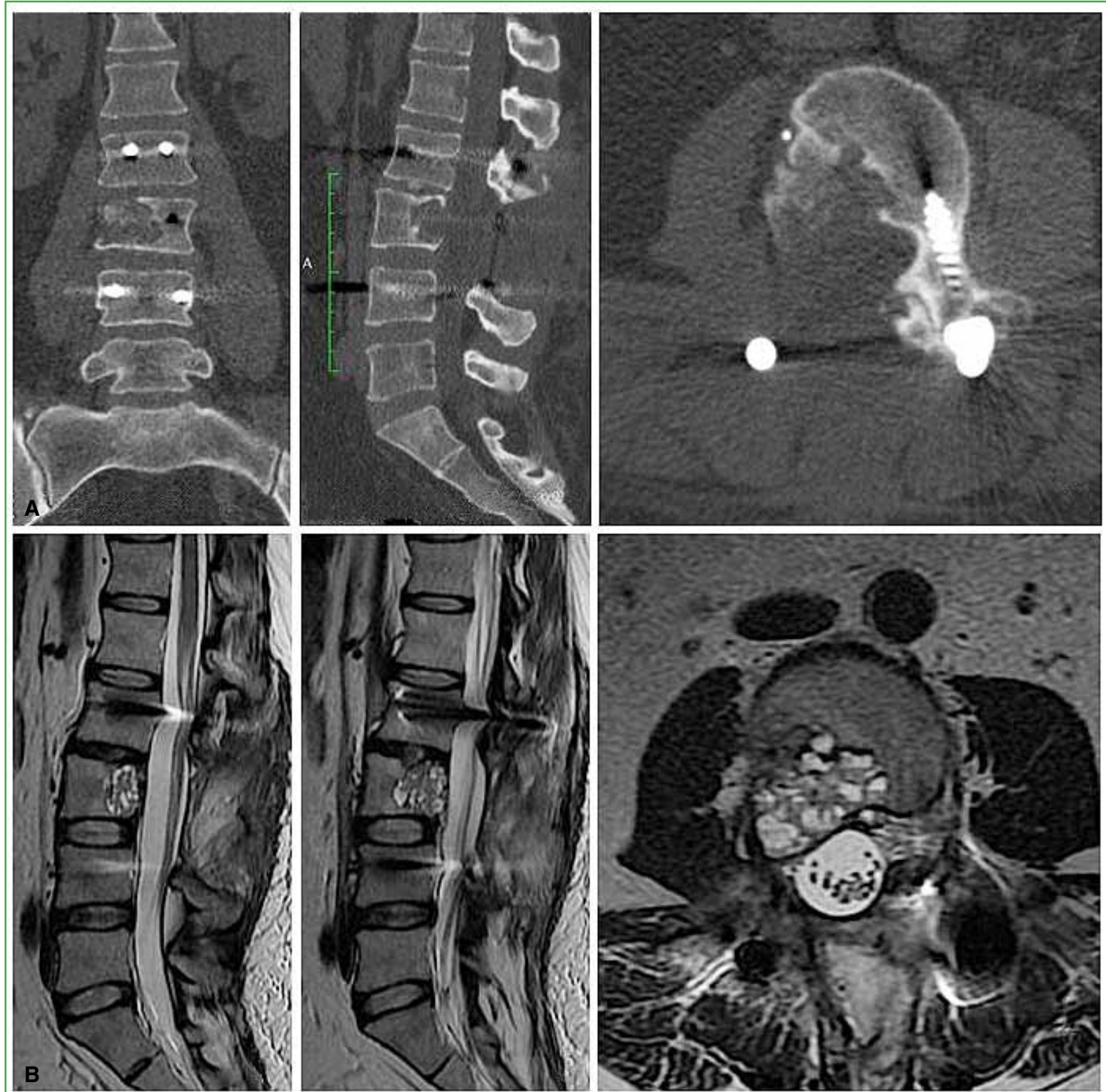

Figura 4. Imágenes de la tomografía computarizada (A) y la resonancia magnética (B) a los seis meses de la cirugía. Se observa la recidiva del tumor. 


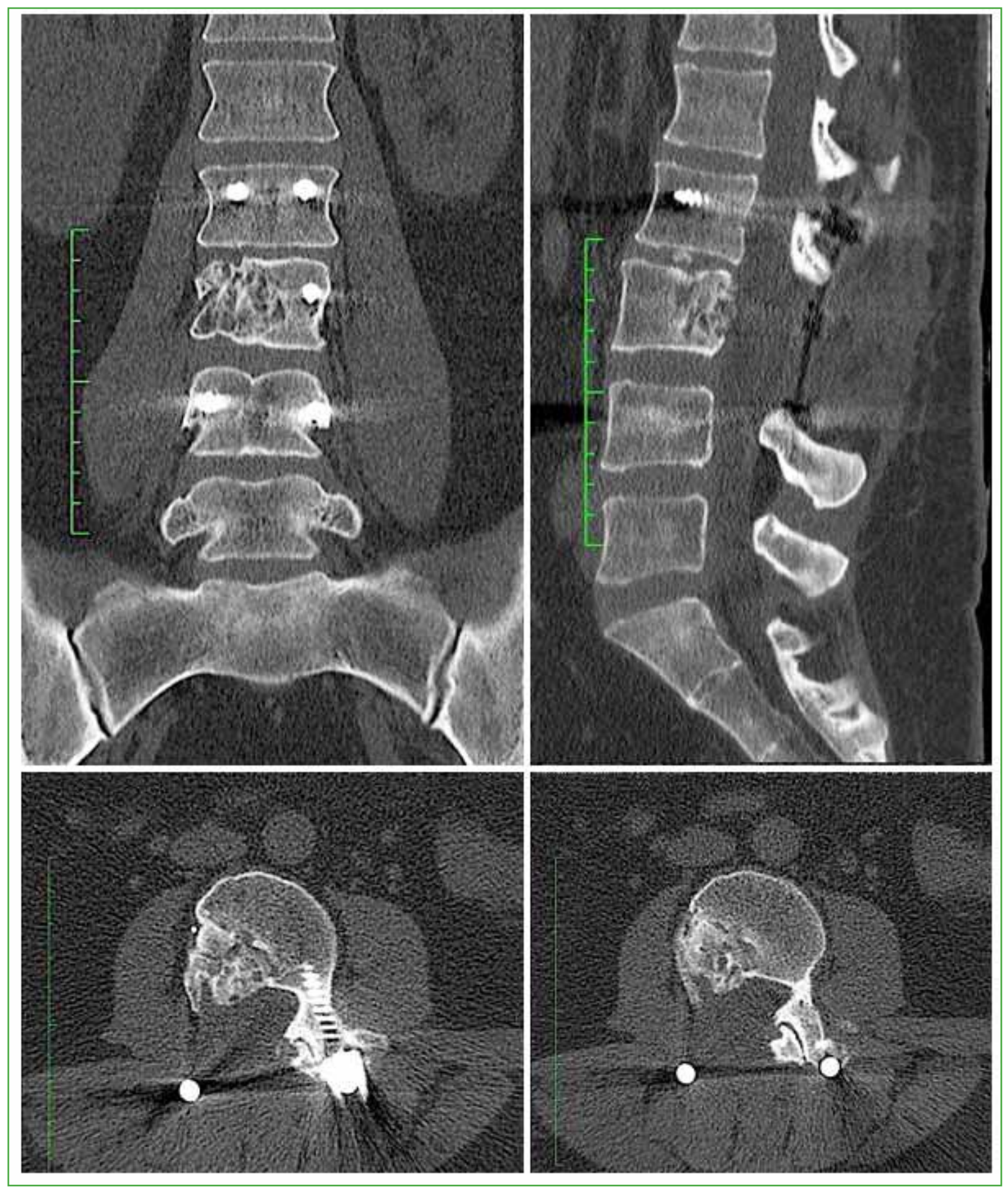

Figura 5. Tomografía computarizada de control después del año de tratamiento con denosumab. 


\section{DISCUSIÓN}

En la actualidad, se discute el uso de denosumab para el tratamiento inicial de cuadros tumorales, pero sería una posibilidad terapéutica para tumores ubicados en áreas de difícil acceso para las técnicas quirúrgicas ${ }^{1,7,9} \mathrm{o}$ inclusive para recidivas locales del QOA, pues se ha demostrado que permite la formación de hueso y la regresión tumoral, ${ }^{7-9}$ lo que se traduce en la disminución o remisión del cuadro clínico del paciente y la mejora radiológica del tumor. La eficacia máxima del tratamiento se logra a los seis meses. ${ }^{9} \mathrm{Si}$ bien los resultados con denosumab son auspiciosos en la actualidad, como en nuestro caso, es preciso tener en cuenta que la eficacia de este fármaco se ha demostrado en estudios preliminares, y aun así los datos sobre la seguridad a largo plazo, las recurrencias y la duración óptima a largo plazo no son concluyentes; por lo tanto, se necesitan ensayos con más casos, estudios longitudinales y clínicos que avalen la duración del tratamiento y la consistencia del denosumab en el QOA. 3,6,8-10

\section{CONCLUSIONES}

La administración de denosumab $120 \mathrm{mg}$ como dosis inicial, y luego una dosis cada tres meses, hasta los dos años resultó beneficiosa para nuestra paciente con una recidiva local después de la embolización y la exéresis tumoral, se logró la remisión total y la calcificación. El uso de este fármaco sigue siendo controvertido antes de una cirugía, ya que no se dispone de evidencia suficiente de pacientes tratados de esa manera.

Conflicto de intereses: Los autores no declaran conflictos de intereses.

ORCID de F. Lanari Zubiaur: https://orcid.org/0000-0003-4030-0959

ORCID de A. O. Godoy Adaro: https://orcid.org/0000-0001-8018-9197

\section{BIBLIOGRAFÍA}

1. Terzi S, Gasbarrini A, Fuiano M, Barbanti Brodano G, Ghermandi R, Bandiera S, et al. Efficacy and safety of selective arterial embolization in the treatment of aneurysmal bone cyst of the mobile spine. Spine 2017;42(15):1130-8. https://doi.org/10.1097/BRS.0000000000002017

2. Ghermandi R, Terzi S, Gasbarrini A, Boriani S. Denosumab: non-surgical treatment option for selective arterial embolization resistant aneurysmal bone cyst of the spine and sacrum. Case report. Eur Rev Med Pharmacol Sci 2016;20(17):3692-5. PMID: 27649673

3. Lange T, Stehling C, Fröhlich B, Klingenhöfer M, Kunkel P, Schneppenheim R, et al. Denosumab: a potential new and innovative treatment option for aneurysmal bone cysts. Eur Spine J 2013;22(6):1417-22. https://doi.org/10.1007/s00586-013-2715-7

4. Namazi H. Letter regarding "Denosumab: a potential new and innovative treatment option for aneurysmal bone cysts". Eur Spine J 2013;22(10):2342. https://doi.org/10.1007/s00586-013-2951

5. Lange T, Schulte TL. Answer to the Letter to the Editor of H. Namazi concerning "Denosumab: a potential new and innovative treatment option for aneurysmal bone cysts" by Lange T, Stehling C, Fröhlich B, Klingenhöfer M, Kunkel P, Schneppenheim R, Escherich G, Gosheger G, Hardes J, Jürgens H, Schulte TL (2013), Eur Spine J; 22(6):1417-1422. Eur Spine J 2013;22(10):2343. https://doi.org/10.1007/s00586-013-2956-5

6. Patel RS, Dhamne AC, Kumar, GA, Kumar N. Denosumab: a potential treatment option for aneurysmal bone cyst of the atlas. Eur Spine J 2018;27 (Suppl 3):S494-S500. https://doi.org/10.1007/s00586-018-5528-X

7. Kurucu N, Akyuz C, Ergen FB, Yalcin B, Aydin B, Varan A, et al. Denosumab treatment in aneurysmal bone cyst: evaluation of nine cases. Pediatr Blood Cancer 2018;65(4):e26926. https://doi.org/10.1002/pbc.26926

8. Skubitz KM, Peltola JC, Santos RC, Cheng EY. Response of aneurysmal bone cyst to denosumab. Spine 2015;40(22):E1201-E1204. https://doi.org/10.1097/BRS.0000000000001027

9. Dubory A, Missenard G, Domont J, Court C. Interest of denosumab for the treatment of giant-cells tumors and aneurysmal bone cysts of the spine. About nine cases. Spine 2016;41(11): E654-E660.

https://doi.org/10.1097/BRS.0000000000001350 
10. Charest-Morin R, Boriani S, Fisher CH, Patel S, Kawahara N, Mendel E, et al. Benign tumors of the spine. Has new chemotherapy and interventional radiology changed the treatment paradigm? Spine 2016;41(20):S178-S185. https://doi.org/10.1097/BRS.0000000000001818

11. Bazán PL, Di Falco R, Borri AE, Medina M, Ciccioli NM, Danielle S. The use of denosumab in giant cell tumors in sacrum. Coluna/Columna 2020;19(2):147-9. https://doi.org/10.1590/S1808-18512020190228839 